University of Nebraska - Lincoln

DigitalCommons@University of Nebraska - Lincoln

September 1988

\title{
Two-photon detachment of the negative chlorine ion
}

Tsin-Fu Jiang

University of Nebraska - Lincoln

Anthony F. Starace

University of Nebraska-Lincoln, astarace1@unl.edu

Follow this and additional works at: https://digitalcommons.unl.edu/physicsstarace

Part of the Physics Commons

Jiang, Tsin-Fu and Starace, Anthony F., "Two-photon detachment of the negative chlorine ion" (1988). Anthony F. Starace Publications. 34.

https://digitalcommons.unl.edu/physicsstarace/34

This Article is brought to you for free and open access by the Research Papers in Physics and Astronomy at DigitalCommons@University of Nebraska - Lincoln. It has been accepted for inclusion in Anthony F. Starace Publications by an authorized administrator of DigitalCommons@University of Nebraska - Lincoln. 


\title{
Two-photon detachment of the negative chlorine ion
}

\author{
Tsin- Fu Jiang* \\ Department of Physics and Astronomy, The University of Nebraska, Lincoln, Nebraska 68588-0111 \\ and Institute of Atomic and Molecular Science, Academia Sinica, P.O. Box 23-166, Taipei, Taiwan 107, Republic of China \\ Anthony F. Starace \\ Department of Physics and Astronomy, The University of Nebraska, Lincoln, Nebraska 68588-0111
}

(Received 22 March 1988)

\begin{abstract}
Theoretical calculations of the two-photon detachment cross section of the negative chlorine ion are presented in the energy region from threshold to the single-photon ionization threshold using a transition-matrix method. Detailed analyses are presented of the effects of various kinds of electron correlations, whose net effect is a reduction of the uncorrelated Hartree-Fock model cross sections by up to $30 \%$. Our cross sections have a much flatter variation with energy and a much lower magnitude (by up to a factor of 2 or 3 ) than the frozen-core free-electron results of M. Crance [J. Phys. B 20, 6553 (1987)] or the central-potential model calculations of E. J. Robinson and S. Geltman [Phys. Rev. 153, 4 (1967)].
\end{abstract}

\section{INTRODUCTION}

The role of electron-correlation effects in nonresonantmultiphoton-ionization (MPI) processes is of interest because of recent experiments on MPI of rare gases and alkaline earth atoms which have revealed unexpectedly intense production of multiply charged ions by strong laser fields. ${ }^{1}$ In the past two years new theoretical studies have been carried out for two-photon ionization of two of the heavier rare gas atoms: $\operatorname{argon}^{2}$ and xenon. ${ }^{3,4}$ In agreement with an earlier study for argon, ${ }^{5}$ these calculations ${ }^{1-4}$ have found the following: The choice of finalstate continuum wave functions is very important, the best being $L S$-dependent Hartree-Fock continuum wave functions; initial-state correlations play a modest role (i.e., they produce 10-15\% corrections in the two-photon cross sections), in contrast to their more significant role in single-photon ionization; and interchannel interactions in the intermediate states are significant mainly in producing the correct resonance structure in the cross sections.

The more recent calculations ${ }^{2-4}$ find in addition a much more significant effect of electron correlations than any of the others mentioned so far. Specifically, they find that there is strong competition between two mechanisms of ionization: the first is that in which each of the two photons acts on the same photoelectron; the second is that in which each of the photons excites a different photoelectron and these two photoelectrons then scatter from one another in such a way that one is de-excited back to its initial state and the other goes off as the single, observable photoelectron. The latter mechanism has been found to reduce the two-photon cross section obtained for the first mechanism by a factor of 2 or more below the one-photon ionization threshold ${ }^{2-4}$ and to produce a corresponding increase above the one-photon ionization threshold. ${ }^{3,4}$ The importance of this second ion- ization mechanism may be understood qualitatively by observing that the coupling of the radiation field with the filled (or nearly filled) outer subshell of bound electrons in the atom (or in the ion) is stronger than the coupling of the radiation field with a single excited photoelectron.

In this paper we extend our transition-matrix calculations for argon to the isoelectronic negative ion of chlorine. While the rare gases are important test cases for theoretical treatments (due in part to the simplicity their spherical symmetry affords and in part to the large number of both theoretical and experimental studies of single photon processes involving them), their large binding energies limit experimental studies to high-order multiphoton processes. In contrast, the isoelectronic negative halogen ions have much smaller binding energies, which permit experimental studies of two- or threephoton ionization processes. Indeed the first relative measurements of the two-photon ionization cross section for the negative chlorine ion have just recently appeared. ${ }^{6}$ Furthermore, new calculations, not including electron correlation effects, of multiphoton detachment from negative halogen ions have just been carried out. ${ }^{7}$ For these reasons it is of great interest to find out what role electron correlations play in the negative halogen ions. Our results here for the negative chlorine ion complement concurrent results ${ }^{8}$ for the negative iodine ion.

In Sec. II we review the basic equations of our transition-matrix approach. ${ }^{2}$ In Sec. III we present our results for each of the three allowed final-state channels as well as for the total cross sections for both linearly and circularly polarized light. We also compare our results with the recent independent electron results of Crance ${ }^{7}$ as well as with the much older results of Robinson and Geltman. ${ }^{9}$ In order to make a proper comparison with the latter calculations, we have investigated in part the role of atomic polarization. Finally, in Sec. IV we present some conclusions. A preliminary report of the present results has been given elsewhere. ${ }^{10}$ 


\section{THEORETICAL PROCEDURE}

The transition-matrix method ${ }^{11,12}$ was applied to twophoton ionization of argon in Ref. 2. The numerical results of that calculation ${ }^{2}$ showed that a simpler theoretical treatment is possible. We focus here on the key approximations made in the present calculation and the final radial equations obtained and refer the reader to these earlier works ${ }^{2,11,12}$ for a more detailed exposition.

\section{A. Implicit summation procedure}

In a two-photon ionization process the transition amplitude $T(\omega)$ for photons of energy $\omega$ between an initial state $i$ and a final state $f$ may be written as

$$
T(\omega)=\sum_{m} \frac{\langle f|D| m\rangle\langle m|D| i\rangle}{\omega+\varepsilon_{i}-\varepsilon_{m}} .
$$

Here $m$ is an intermediate atomic state of energy $\varepsilon_{m}$ and $D$ is the electric dipole operator. Dalgarno and Lewis ${ }^{13}$ defined the effective function $\lambda(\omega)$, where

$$
|\lambda(\omega)\rangle=\sum_{m} \frac{|m\rangle\langle m|D| i\rangle}{\omega+\varepsilon_{i}-\varepsilon_{m}},
$$

and showed that $\lambda(\omega)$ may be obtained as the solution of the following inhomogeneous differential equation:

$$
\left(\omega+\varepsilon_{i}-H\right)|\lambda(\omega)\rangle=D|i\rangle .
$$

Then $T(\omega)$ is simply given by

$$
T(\omega)=\langle f|D| \lambda(\omega)\rangle .
$$

The initial and final states, $i$ and $f$, respectively, are presumed to be eigenstates of the exact Hamiltonian $H$, with eigenvalues $\varepsilon_{i}$ and $\varepsilon_{f}$, where $\varepsilon_{f}=\varepsilon_{i}+2 \omega$. The main task of any theory of two-photon ionization is then to specify representations for the initial, intermediate, and final states, $i, \lambda$, and $f$, respectively, appearing in Eqs. (3) and (4) and to derive solvable equations for any unknown functions appearing in these representations that are needed for the calculation of Eq. (4).

\section{B. The transition-matrix method}

Motivation for a transition-matrix approach stems from the observation that the dipole operator in Eq. (4) is a one-electron operator, i.e.,

$$
D \equiv \hat{\boldsymbol{\epsilon}} \cdot \sum_{i=1}^{N} \mathbf{r}_{i}
$$

where $\epsilon$ is the polarization of the photons and $\mathbf{r}_{i}$ is the radial coordinate of the $i$ th atomic electron $(1 \leq i \leq N)$. Hence, the transition amplitude $T(\omega)$ in Eq. (4) may be expressed in terms of one-electron functions for the atomic states. These one-electron functions are obtained in the transition-matrix method ${ }^{2,11,12}$ by integrating the following commutator equation for the outer product $|\lambda(\omega)\rangle\langle f|$ over the first $N-1$ electron coordinates:

$$
[H,|\lambda(\omega)\rangle\langle f|]=-\omega|\lambda(\omega)\rangle\langle f|-D| i\rangle\langle f| .
$$

One must assume, of course, a particular form for the states $i, \lambda(\omega)$, and $f$.

In this work we represent the initial, intermediate, and final states as follows:

$$
\begin{aligned}
|i\rangle= & \left|3 p^{6}\left({ }^{1} S\right)\right\rangle \\
& +\sum_{\bar{L} \bar{S}} \sum_{l=2}^{3} b_{l}^{i}(\bar{L} \bar{S})\left|3 p^{4}(\bar{L} \bar{S}) \phi_{l}^{i} \phi_{l}^{i}(\bar{L} \bar{S})\left({ }^{1} S\right)\right\rangle \\
|\lambda(\omega)\rangle= & \sum_{l_{\lambda}=0,2}\left|3 p^{5} \psi_{l_{\lambda}}^{\lambda}\left({ }^{1} P\right)\right\rangle \\
|f\rangle= & \mid 3 p^{5} \psi_{l_{f}}^{f}\left({ }^{1} L\right) .
\end{aligned}
$$

The $\mathrm{Cl}^{-}$ground state $|i\rangle$ is thus represented by an admixture of the normal $3 p^{6}$ configuration plus a sum of doubly excited configurations in which two $3 p$ electrons are virtually excited to a pair of $d$ or $f$ orbitals. The intermediate state $|\lambda(\omega)\rangle$ is represented by a singly excited state, in which one $3 p$ electron is photoexcited to the $\psi_{l_{\lambda}}^{\lambda}\left(l_{\lambda}=0\right.$ or 2$)$ orbital. We ignore doubly excited configurations in the representation for $|\lambda(\omega)\rangle$ arising from photoexcitation of the doubly excited configurations in the representation for $|i\rangle$ since these were found to have negligible contributions to the two-photon cross section in our calculations for argon. ${ }^{2}$ This approximation significantly simplifies the present calculation. Our final state is assumed to have only one electron excited out of the $3 p$ subshell. This final-state electron's orbital angular momentum $l_{f}$ is 1 or 3 . Due to angular momentum selection rules for absorption of two photons having the same polarization, the $L=1$ final state is not allowed.

\section{Two-photon transition amplitude}

The desired transition amplitude $T(\omega)$ is calculated by substituting the states in Eq. (7) in Eq. (4) to obtain

$$
\begin{aligned}
T(\omega)= & \left(\psi_{l_{f}}^{f}|r| \psi_{l_{\lambda}}^{\lambda}\right)\left(l_{f}\left\|C^{[1]}\right\| l_{\lambda}\right)[1]^{1 / 2}[L]^{1 / 2} \\
& \times\left\{\begin{array}{lll}
l_{\lambda} & 1 & 1 \\
L & l_{f} & 1
\end{array}\right\} .
\end{aligned}
$$

The reduced angular matrix element in Eq. (8) is defined by

$$
\left(a\left\|C^{[k]}\right\| b\right) \equiv(-1)^{a}[a]^{1 / 2}[b]^{1 / 2}\left(\begin{array}{lll}
a & k & b \\
0 & 0 & 0
\end{array}\right),
$$

where the 6 numbers in braces represent a 3-j symbol and $[a] \equiv 2 a+1$. The radial dipole matrix element is defined by

$$
\left(\psi_{a}|r| \psi_{b}\right) \equiv \int_{0}^{\infty} r^{2} d r \psi_{a}^{*}(r) r \psi_{b}(r)
$$

\section{Equations for one-electron radial functions}

Calculation of the desired two-photon transition amplitude in Eq. (8), therefore, requires only that we obtain the one-electron radial functions $\psi_{l_{\lambda}}^{\lambda}(r), \psi_{l_{f}}^{f}(r)$ and $\chi_{3 p}(r)$. 
We do this by substituting the representations for the initial, intermediate, and final states given in Eq. (7) into the equation of state in Eq. (6), integrating over the first $N-1$ electron spatial and spin coordinates, keeping only particle-hole interactions, and equating to zero the coefficients of certain double-tensor operators as described in detail in Refs. 11 and 12 . The resulting differential equation for $\psi_{l_{\lambda}}^{\lambda}(r)$ is

$$
\begin{aligned}
& \left(-\frac{1}{2} \frac{d^{2}}{d r^{2}}-\frac{Z}{r}+\frac{1}{2} \frac{l(l+1)}{r^{2}}-\left(\omega+\varepsilon_{3 p}\right)\right) \psi_{l}^{\lambda}(r) \\
& +\sum_{n_{1} l_{1}}^{\neq 3 p}\left[\left(l\left\|\boldsymbol{V}^{0}\left(n_{1} l_{1}, n_{1} l_{1} ; r\right)\right\| l\right) 2 \frac{\left[l_{1}\right]^{1 / 2}}{[l]^{1 / 2}} \psi_{l}^{\lambda}(r)+\sum_{k}(-1)^{k+1}\left(l\left\|\boldsymbol{V}^{k}\left(n_{1} l_{1}, \psi_{l}^{\lambda} ; r\right)\right\| l_{1}\right) \frac{1}{[l]} \chi_{n_{1} l_{1}}(\boldsymbol{r})\right] \\
& +\sum_{l^{\prime}}\left[\left(l\left\|V^{k}(3 p, 3 p ; r)\right\| l^{\prime}\right)\left(2 \frac{[1]^{1 / 2}}{[l]^{1 / 2}} \delta_{l l^{\prime}} \delta_{k 0}-\left\{\begin{array}{ccc}
1 & 1 & k \\
l^{\prime} & l & 1
\end{array}\right\}\right) \psi_{l^{\prime}}^{\lambda}(r)+\left(l\left\|V^{k}\left(3 p, \psi_{l}^{\lambda} ; r\right)\right\| 1\right)\left(-\frac{2}{3} \delta_{k 1}+\frac{\delta_{l^{\prime} l}}{[l]}\right] \chi_{3 p}(r)\right] \\
& =+2^{1 / 2}\left(l\left\|C^{[1]}\right\| 1\right) \chi_{3 p}(r) r+\sum_{\bar{L}, \bar{S}}[(2)(3)(5)]^{1 / 2}\left(3 p^{52} P\left\{\mid 3 p^{4} \bar{L} \bar{S}\right) b_{l}^{i}(\bar{L} \bar{S})\left(1\left\|C^{[1]}\right\| l\right)\right. \\
& \times\left(\chi_{3 p}|r| \phi_{l}^{i}\right)(-1)^{\bar{L}}\left\{\begin{array}{ccc}
l & l & \bar{L} \\
1 & 1 & 1
\end{array}\right\} \phi_{l}^{i}(r) .
\end{aligned}
$$

In Eq. (11) the following reduced matrix element resulting from the Coulomb interaction has been defined: ${ }^{11}$

$$
\left(l_{2}\left\|V^{k}\left(n_{1} l_{1}, n_{3} l_{3} ; r\right)\right\| l_{4}\right) \equiv \int_{0}^{\infty} d r \frac{r_{<}^{k}}{r_{>}^{k+1}} P_{n_{1} l_{1}}(r) P_{n_{3} l_{3}}(r)\left(l_{1}\left\|C^{[k]}\right\| l_{3}\right)\left(l_{2}\left\|C^{[k]}\right\| l_{4}\right) \text {. }
$$

Note that the first term on the right-hand side in Eq. (11), proportional to $r \chi_{3 p}(r)$, is the usual source term for populating the intermediate excited state $\psi_{l}^{\lambda}(r)$, namely, a dipole transition of a $3 p$-subshell electron to a state of angular momentum $l$. This term stems from the usual $3 p^{6}$ configuration of the negative chlorine ion ground state [cf. the first term of Eq. (7a)]. The second term on the right-hand side of Eq. (11), proportional to $\left(\chi_{3 p}|r| \phi_{l}^{i}\right) \phi_{l}^{i}(r)$, arises from the virtual doubly excited states in the negative chlorine ion ground state [cf. the second term of Eq. (7a)]. In this case the first photon deexcites one of the doubly excited electrons, described by $\phi_{l}^{i}$, to the $3 p$ subshell with an amplitude proportional to $\left(\chi_{3 p}|r| \phi_{l}^{i}\right)$. The other doubly excited electron, also described by $\phi_{l}^{i}$, then is left to contribute to the amplitude $\psi_{l}^{\lambda}(r)$. Note that since $\psi_{l}^{\lambda}(r)$ can only have $l=0$ or 2 (by dipole selection rules) and since $\phi_{l}^{i}(r)$ is assumed to have $l$ values of 2 or 3 , it is obvious that only $\phi_{d}^{i}(r)$ contributes to $\psi_{d}^{\lambda} ; \phi_{f}^{i}(r)$ plays no role here other than to reduce the amplitudes of the other configurations in the ground state. Finally, we indicate how the other radial functions needed to calculate Eq. (8) and to solve Eq. (11) are obtained.

First, as is the case in single-photon ionization, we assume that the radial functions $\chi_{n l}(r)$ for the bound $n l$ electron are obtained from a Hartree-Fock (HF) calculation for the ${ }^{1} S$ ground state of the negative chlorine ion.

Second, the final-state radial function $\psi_{l_{f}}^{f}(r)$ is obtained from an $L S$-dependent HF calculation in the field of the unrelaxed chlorine atom having an outer $p^{5}$ subshell. The radial equation is similar in form to that for $\psi_{l_{\lambda}}^{\lambda}(r)$ in Eq. (11) except for the absence of source terms.

Third, the initial-state correlation functions $\phi_{l}^{i}$ $(l=2,3)$ may be obtained using the multiconfiguration HF code of Froese-Fischer. ${ }^{14}$ The use of an average function $\phi_{l}^{i}$ instead of functions dependent on the term level, $\bar{L} \bar{S}$, of the $p^{4}$ core [cf. Eq. (7a)] has been found to be a good approximation in a single-photon ionization study of rare gases. ${ }^{15}$

\section{E. Electron-scattering interactions}

Until very recently, all calculations for two-photon one-electron ionization processes restricted their treatment of electron correlations to those in which only one of the two photons interacts with the target and the other photon interacts with one of the electrons excited from the target. Our transition-matrix approach, described above, also employs this restriction. (Our approach can treat a more general class of interactions, but the calculation of interactions between atomic states having several electrons excited from the target quickly becomes rather complex.) L'Huillier et al. ${ }^{16}$ approximately treated in addition to these interactions a set of electron correlations in which each photon excites an electron from the unexcited atomic target. The electrons then interact in such a way that one electron is de-excited back to the core and the other goes off as the single observable photo- 
electron. These interactions were found to reduce the two-photon single ionization cross sections of both argon $^{2}$ and xenon ${ }^{3,4}$ by a large fraction in the region below the single-photon ionization threshold. In the region above the single-photon ionization threshold in xenon, ${ }^{3,4}$ these interactions were found to increase the cross sections significantly.

These interactions are specified exactly (in lowest order) by the many-body perturbation theory (MBPT) diagrams shown in Figs. 1(a) and 1(b). Each of the diagrams in Fig. 1 describes a particular path by which the two photons ionize a single electron from the $n_{0} l_{0}$ subshell. It is intuitively clear why these interactions turn out to be important. One knows in the case of rare gas atoms that the single-photon electric dipole transition from the outer $p^{6}$ subshell is very strong, particularly above the singlephoton ionization threshold. ${ }^{17,18}$ The diagrams in Fig. 1 introduce this interaction twice, once for each photon. Roughly speaking, this transition amplitude is stronger for an $n_{0} l_{0}$ electron than for an excited electron by a factor corresponding to the occupation number of the $n_{0} l_{0}$ subshell; however, potential barrier effects important for the transition $n_{0} l_{0} \rightarrow \epsilon\left(l_{0}+1\right)$ come into play as well.

Exact evaluation of the interactions in Fig. 1 by the transition-matrix approach is complex. The doubly excited intermediate states introduce complicated angular momentum algebra and summations over coefficients of fractional parentage. Previous calculations for argon ${ }^{2}$ and for xenon ${ }^{3,4}$ treated the interactions in Fig. 1 by means of the random-phase approximation (RPA): Each interaction of a photon with the $n_{0} l_{0}$ subshell is treated as if it were a transition of the type $n_{0} l_{0}^{4 l_{0}+2}\left({ }^{1} S\right)+\gamma \rightarrow n_{0} l_{0}^{4 l_{0}+1} \epsilon l\left({ }^{1} P\right)$, i.e., a single-photon ionization process from the ground configuration, in which the transition amplitude used is the RPA amplitude.

In this approximation, the transition amplitude in Eq. (8) is modified to $^{2}$

$$
\begin{aligned}
T(\omega)= & \left(l_{f}\left\|C^{[1]}\right\| l_{\lambda}\right)[1]^{1 / 2}[L]^{1 / 2}\left\{\begin{array}{ccc}
l_{\lambda} & 1 & 1 \\
L & l_{f} & 1
\end{array}\right\} \\
& \times\left[\left(\psi_{l_{f}}^{f}|r| \psi_{l_{\lambda}}^{\lambda}\left(\omega+\varepsilon_{3 p}\right)\right)-\left(3^{-1 / 2} \sum_{l_{\lambda}^{\prime}}\left(1\left\|C^{[1]}\right\| l_{\lambda}^{\prime}\right) R^{1}\left(\psi_{l_{f}}^{f}, \chi_{3 p} ; \psi_{l_{\lambda}}^{\lambda}\left(\omega+\varepsilon_{3 p}\right), \psi_{l_{\lambda}^{\prime}}^{\lambda}\left(\omega+\varepsilon_{3 p}\right)\right)\right]\right. \\
& \left.-\left(3^{-1 / 2} \sum_{l_{\lambda}^{\prime}}\left(l_{\lambda}^{\prime}\left\|C^{[1]}\right\| 1\right) R^{1}\left(\psi_{l_{f}}^{f}, \psi_{l_{\lambda}^{\prime}}^{\lambda}\left(\varepsilon_{3 p}-\omega\right) ; \psi_{l_{\lambda}}^{\lambda}\left(\omega+\varepsilon_{3 p}\right), \chi_{3 p}\right)\right]\right)
\end{aligned}
$$

In Eq. (13) the second sum in large parentheses describes the interaction in Fig. 1(a) while the first sum in large parentheses describes the interaction shown in Fig. 1(b) as well as the corresponding interaction in which the time ordering of the photons is reversed. Also in Eq. (13), generalized Slater integrals ${ }^{19}$ are indicated by $R^{1}$, and the energy at which each $\psi^{\lambda}$ is calculated is indicated in parentheses [cf. Eq. (11)].

Note that interactions of higher order than shown in Fig. 1 are included implicitly in our expression for the transition amplitude in Eq. (13). Due to the second source term on the right-hand side of Eq. (11) above, the effects of electron pairs virtually excited out of the ground state are included also in describing the photonatom interaction. Such higher-order effects are included also in Refs. 3, 4, and 14, where they are included as part of the "screened interaction" described there.

\section{RESULTS AND DISCUSSIONS}

We are concerned with the calculation of the cross sections for each of the following transitions:

$$
\begin{aligned}
\mathrm{Cl}^{-} 3 p^{6}\left({ }^{1} S\right)+2 \gamma & \rightarrow \mathrm{Cl} 3 p^{5}\left({ }^{2} P\right) \epsilon f\left({ }^{1} D\right) \\
& \rightarrow \mathrm{Cl} 3 p^{5}\left({ }^{2} P\right) \epsilon p\left({ }^{1} D\right) \\
& \rightarrow \mathrm{Cl} 3 p 5\left({ }^{2} P\right) \epsilon p\left({ }^{1} S\right)
\end{aligned}
$$

We first discuss some numerical details of our calculations and then present our cross-section results for each of these three channels as well as for the total cross section.

\section{A. Numerical details}

Our initial state is represented as a superposition of the frozen-core Hartree-Fock (HF) ground-state configuration $3 p^{6}\left({ }^{1} S\right)$ and configurations having pairs of $d$ and $f$ electrons excited out of the ground-state configuration. As shown by Froese-Fischer, ${ }^{14}$ such excited pairs of electrons may be well represented by a diagonal expansion of the form $3 d^{2}, 4 d^{2}, \ldots, 4 f^{2}, 5 f^{2}, \ldots$ Furthermore, Swanson and Armstrong ${ }^{15}$ have found that in the case of argon these expansions are so rapidly convergent that only the leading terms need to be included. We have therefore included only the $3 d^{2}$ and $4 f^{2}$ excited pairs in our representation for the initial state. We have used the MCHF computer program of Froese-Fischer ${ }^{20}$ to calculate firstly the $\mathrm{Cl}^{-} 3 p^{6}\left({ }^{1} \mathrm{~S}\right)$ single configuration groundstate wave functions. These were then used as "frozen" input orbitals to a multiconfiguration calculation that mixed the $3 p^{6}$ configuration with configurations of the type $3 p^{4} 3 d^{2}$ and $3 p^{4} 4 f^{2}$. The calculated weights of each of these configurations are given in Table I. Note that only a single $3 d$ orbital wave function and a single $4 f$ or- 


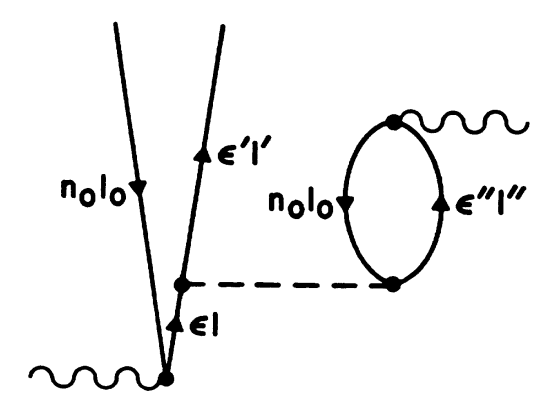

(a)

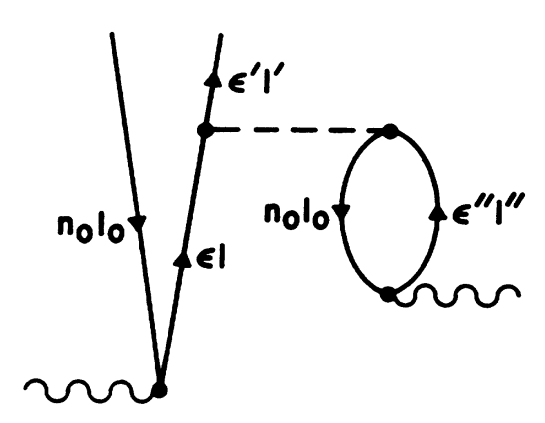

(b)

FIG. 1. Many-body perturbation theory diagrams for the electron scattering interactions relevant to two-photon ionization processes. See text for description.

bital wave function were calculated; i.e., as in Ref. 15, the term dependence of these orbitals was ignored.

The final-state wave function for the photoelectron in each of the configurations on the right-hand side in Eq. (14) was calculated in the $V^{N-1}(L S)$ potential ${ }^{21}$ for the ion. We employed the frozen core approximation, i.e., the one-electron orbitals of the $\mathrm{Cl} 3 p^{5}\left({ }^{2} P\right)$ configuration were the same as those calculated for the $\mathrm{Cl}^{-} 3 p^{6}\left({ }^{1} S\right)$ initial configuration. Orthogonality of the continuum $p$ electron wave functions to the bound $2 p$ and $3 p$ wave functions was assured by calculating both the bound and continuum orbitals in the same Hermitian potential, as described in Refs. 22-24. Each of our continuum wave functions was energy normalized at large radial distances:

TABLE I. Weights of initial-state configurations.

\begin{tabular}{lr}
\hline \multicolumn{1}{c}{ Configuration } & Weight $^{\mathrm{a}}$ \\
\hline $\mathrm{Cl}^{-} 3 p^{6}\left({ }^{1} S\right)$ & 0.9762 \\
$\mathrm{Cl}^{-} 3 p^{4}\left({ }^{1} S\right) 3 d^{2}\left({ }^{1} S\right)$ & 0.1127 \\
$\mathrm{Cl}^{-} 3 p^{4}\left({ }^{1} D\right) 3 d^{2}\left({ }^{1} S\right)$ & 0.1232 \\
$\mathrm{Cl}^{-} 3 p^{4}\left({ }^{3} P\right) 3 d^{2}\left({ }^{1} S\right)$ & 0.1306 \\
$\mathrm{Cl}^{-} 3 p^{4}\left({ }^{1} S\right) 4 f^{2}\left({ }^{1} S\right)$ & -0.0253 \\
$\mathrm{Cl}^{-} 3 p^{4}\left({ }^{1} D\right) 4 f^{2}\left({ }^{1} S\right)$ & -0.0250 \\
$\mathrm{Cl}^{-} 3 p^{4}\left({ }^{3} P\right) 4 f^{2}\left({ }^{1} S\right)$ & -0.0282 \\
\hline \hline
\end{tabular}

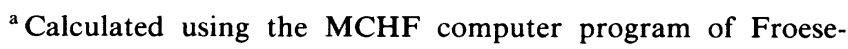
Fischer (cf. Ref. 20). $\psi_{l_{f}}^{f}(k, r)=\left(\frac{2}{\pi \zeta\left(l_{f}, k, r\right)}\right)^{1 / 2} \sin \left[\theta\left(l_{f}, k, r\right)+\delta\right]$,

where

$$
\begin{aligned}
& \theta\left(l_{f}, k, r\right) \underset{r \rightarrow \infty}{\rightarrow} k r-\frac{1}{2} \pi l_{f}, \\
& \zeta\left(l_{f}, k, r\right) \underset{r \rightarrow \infty}{\rightarrow} k .
\end{aligned}
$$

In Eq. (15), $k^{2} / 2$ is the kinetic energy of the photoelectron and $\delta$ is the phase shift. The phase function $\theta\left(l_{f}, k, r\right)$ and the amplitude function $\zeta\left(l_{f}, k, r\right)$ are calculated at large but finite $r$ using the procedure of Burgess. $^{25}$ In our calculations we have ignored the final-state interchannel coupling between the $f^{1} D$ and $p^{1} D$ channels.

The intermediate-state functions $\psi_{l_{\lambda}}^{\lambda}$ which are needed to calculate the transition amplitudes in Eqs. (8) and (13) were obtained as the solutions of Eq. (11). Each of the source terms on the right-hand side in this equation was calculated using the one-electron orbital wave functions calculated for the initial state. Since we have restricted our calculations to the region below the one-photon ionization threshold, $\psi_{l_{f}}^{\lambda}(r)$ satisfies bound state boundary conditions at large $r$. In solving Eqs. (11) we have used the HF value for the $3 p$-orbital energy $\varepsilon_{3 p}$, i.e., $\varepsilon_{3 p}^{\mathrm{HF}}=-0.1503$ a.u., where 1 a.u. $=27.2108 \mathrm{eV}$.

\section{B. Results for the generalized two-photon cross sections}

In lowest-order perturbation theory the $N$-photon ionization rate $W$ is given by ${ }^{26}$

$$
W=\sigma_{N} I^{N}
$$

Here $\sigma_{N}$ is a generalized $N$-photon cross section dependent only on properties of the atomic or molecular target and on the polarization of the incident light and $I$ is the intensity of the laser field. $W$ is usually measured in units of ions/sec, $\sigma_{N}$ in units of $\mathrm{cm}^{2 N} \mathrm{sec}^{N-1}$, and $I$ in units of photons $/\left(\mathrm{cm}^{2} \mathrm{sec}\right)$. For two-photon ionization, the generalized cross section in atomic units (i.e., $e=\hbar=m=1$ ) is

$$
\sigma_{2}^{q}=\frac{8 \pi^{3} \omega^{2}}{c^{2}}|T|^{2} f_{q} \text { (a.u.) }
$$

Here $\omega$ is the photon energy, $c$ is the speed of light, $T$ is the reduced transition-matrix element [cf. Eqs. (8) and (13)], and $f_{q}$ is a geometrical factor dependent on the polarization $q$ of the photons and on the final-state term level. Table II gives the values of $f_{q}$ for the cases of interest in this paper. Conversion of Eq. (17) to the usual units $\mathrm{cm}^{4} \mathrm{sec}$ requires the conversion of the dimension $L^{4} T$ from a.u. to c.g.s. units,

$$
L^{4} T \text { (a.u.) }=1.8967 \times 10^{-50} \mathrm{~cm}^{4} \mathrm{sec} \text {. }
$$

We present our length form results for the generalized two-photon cross section for each of the three transitions in Eq. (14) in the case of linearly polarized photons in Figs. 2-4. In computing the cross sections we employed 
TABLE II. Values for $f_{q}$ [cf. Eq. (17)] for linearly polarized $(q=0)$ and circularly polarized $(q= \pm 1)$ photons.

\begin{tabular}{ccc}
\hline \multicolumn{4}{c}{ Final-state } & \\
$q$ & term level & $f_{q}$ \\
\hline 0 & ${ }^{1} S$ & $\frac{1}{9}$ \\
0 & ${ }^{1} D$ & $\frac{2}{45}$ \\
\pm 1 & ${ }^{1} S$ & 0 \\
\pm 1 & ${ }^{1} D$ & $\frac{1}{15}$ \\
\hline \hline
\end{tabular}

the experimental threshold photon energy $3.613 \mathrm{eV}$. Our results are shown in four levels of approximation, which we discuss in turn. In the Hartree-Fock (HF) approximation we calculate the transition matrix $T(\omega)$ using Eq. (8); the function $\psi_{l_{\lambda}}^{\lambda}$ is computed using only the first source term on the right-hand side of Eq. (11) and ignoring interchannel coupling by requiring $l^{\prime}=l$ in the sum over $l^{\prime}$ in Eq. (11). At the next level of approximation, we include ground-state correlations (GSC's) by calculating the transition matrix $T(\omega)$ using Eq. (8) and computing the function $\psi_{l_{\lambda}}^{\lambda}$ using both source terms on the right-hand side of Eq. (11) and ignoring interchannel coupling by requiring $l^{\prime}=l$ in the sum over $l^{\prime}$ in Eq. (11). In the third level of approximation we include both groundstate correlations and intermediate-state interchannel coupling (IIC): the transition matrix $T(\omega)$ is computed from Eq. (8) and the function $\psi_{l_{\lambda}}^{\lambda}$ from Eq. (11). Lastly, in the fourth level of approximation, we add the effect of the electron scattering interactions (ESI's) by calculating the transition matrix $T(\omega)$ from Eq. (13).

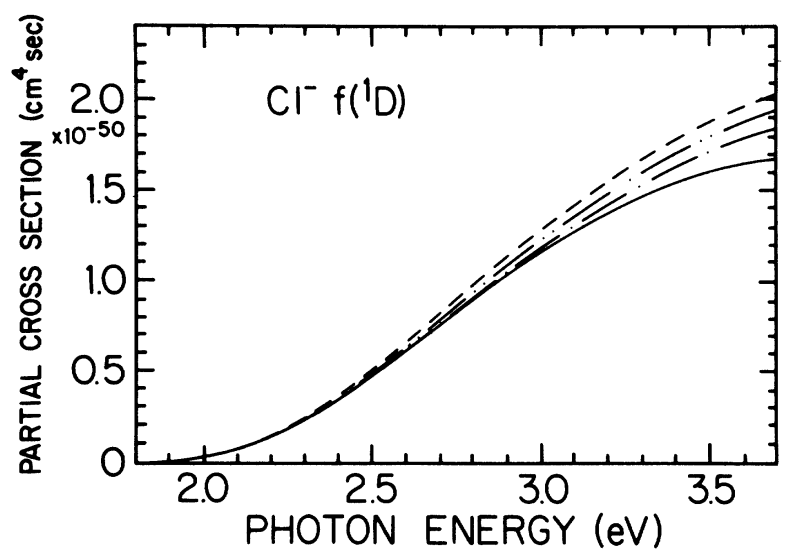

FIG. 2. Generalized two-photon cross section (length form) for the transition $\mathrm{Cl}^{-} 3 p^{6}\left({ }^{1} \mathrm{~S}\right)+2 \gamma \rightarrow \mathrm{Cl} 3 p^{5} \epsilon f\left({ }^{1} D\right)$ for linearly polarized incident photons with energies below the singleionization threshold. Our results are presented in four different approximations, discussed in the text, which are identified as follows:,-- , HF results; $-\cdot-\cdot-\cdot$, HF plus ground-state correlations (GSC's); - - - , HF plus GSC plus intermediatestate interchannel coupling (IIC); - , HF plus GSC plus IIC plus electron scattering interactions (ESI's).

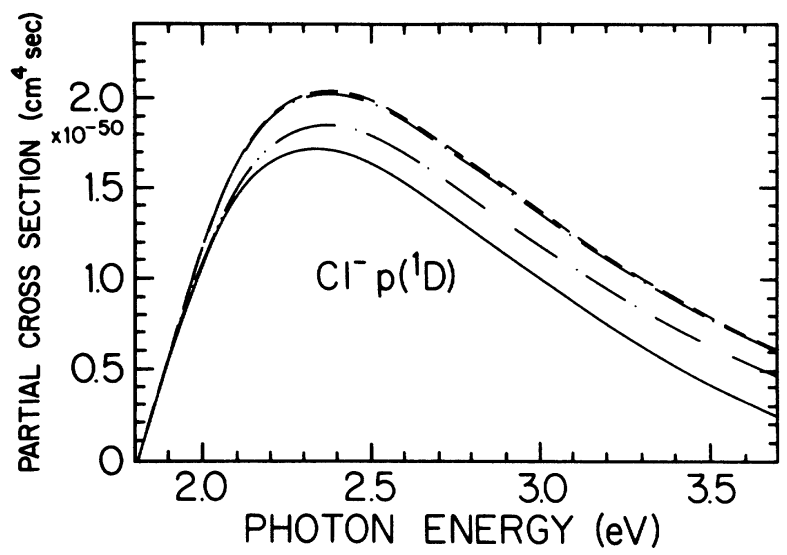

FIG. 3. Generalized two-photon cross section (length form) for the transition $\mathrm{Cl}^{-} 3 p^{6}\left({ }^{1} S\right)+2 \gamma \rightarrow \mathrm{Cl} 3 p^{5} \epsilon p\left({ }^{1} D\right)$ for linearly polarized incident photons with energies below the singleionization threshold. Notations are the same as for Fig. 2.

In comparing Fig. 2 with Figs. 3 and 4 we observe the much smaller magnitude of the $f\left({ }^{1} D\right)$ partial cross section above threshold as compared to either the $p\left({ }^{1} D\right)$ or the $p\left({ }^{1} S\right)$ partial cross sections. This is an example of the well-known Wigner threshold law ${ }^{27}$

$$
\sigma \propto k^{2 l+1},
$$

where in this case $\sigma$ is the generalized two-photon cross section, $k$ is the wave number of the photoelectron, and $l$ is its final-state orbital angular momentum. This result may be understood as an effect of the angular momentum potential repelling higher $l$ states. ${ }^{28}$

In comparing the effects of correlations in each of the three channels we observe a number of both similarities and differences. Firstly, electron correlation effects are minimal just at threshold in all channels. However, while correlation effects begin to be significant by about $0.2 \mathrm{eV}$ above threshold in the case of the $p\left({ }^{1} D\right)$ and $p\left({ }^{1} S\right)$ chan-

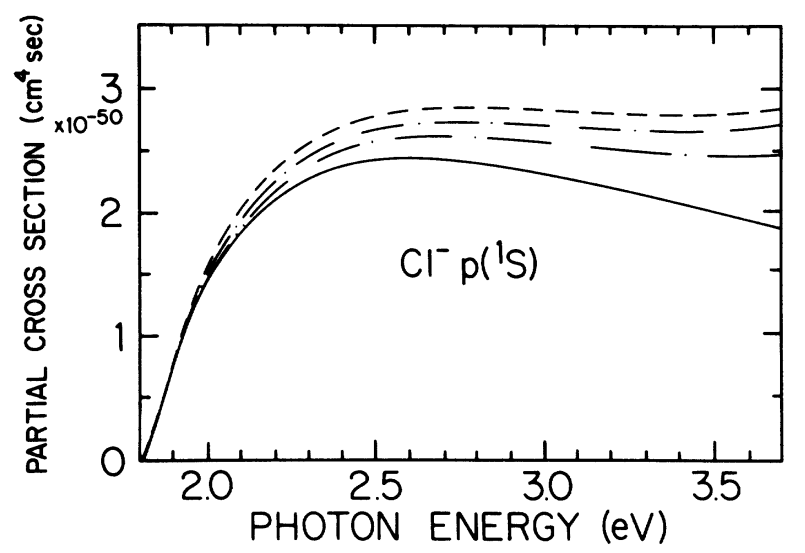

FIG. 4. Generalized two-photon cross section (length form) for the transition $\mathrm{Cl}^{-} 3 p^{6}\left({ }^{1} S\right)+2 \gamma \rightarrow \mathrm{Cl} 3 p^{5} \epsilon p\left({ }^{1} S\right)$ for linearly polarized incident photons with energies below the singleionization threshold. Notations are the same as for Fig. 2. 
nels, they only become significant beyond $0.5 \mathrm{eV}$ above threshold in the case of the $f\left({ }^{1} D\right)$ channel. Secondly, in all channels the HF cross sections are largest, the ESI cross sections are smallest, and the GSC and IIC cross sections lie in between. However, whereas the GSC cross sections lie above the IIC cross sections in the cases of the $p\left({ }^{1} D\right)$ and $p\left({ }^{1} S\right)$ channels, this situation is reversed in the case of the $f\left({ }^{1} D\right)$ channel, implying a net cancellation of ground state and interchannel coupling effects in this latter case. Lastly, while the electron scattering interactions are the dominant corrections for the $p\left({ }^{1} D\right)$ and $p\left({ }^{1} S\right)$ channels, both in absolute magnitude and in percent, these interactions have a much more modest effect on the $f\left({ }^{1} D\right)$ partial cross section. However, the increase in the size of the ESI corrections for the $p$ channels with increasing photon energy, particularly after the curves "turn over" around $2.3 \mathrm{eV}$, leads one to expect a more significant influence of the ESI interactions on the $f\left({ }^{1} D\right)$ channel cross section at photon energies greater than the single-photon ionization threshold.

The total generalized two-photon cross sections for both linearly polarized and circularly polarized light are shown in Figs. 5 and 6, respectively. The total cross section for linearly polarized light is simply the sum of the three partial cross sections shown in Figs. 2-4. The total cross section for circularly polarized light is simply a factor 1.5 times the sum of the $f\left({ }^{1} D\right)$ and the $p\left({ }^{1} D\right)$ cross sections in Figs. 2 and 3, i.e., the $p\left({ }^{1} S\right)$ cross section does not contribute (cf. Table II). In both cases we see that the sharp drop in the $p\left({ }^{1} D\right)$ partial cross section with increasing photon energy is almost completely compensated by the sharply rising $f\left({ }^{1} D\right)$ cross section over the same energy region. Interestingly, this decline is only noticeable in the solid curves, which represent the calculations having the most complete treatment of electron correlation effects. Furthermore, this decline is considerably more noticeable in the cross section for linearly polarized photons than in that for circularly polarized photons. This fact opens the possibility for experiment to

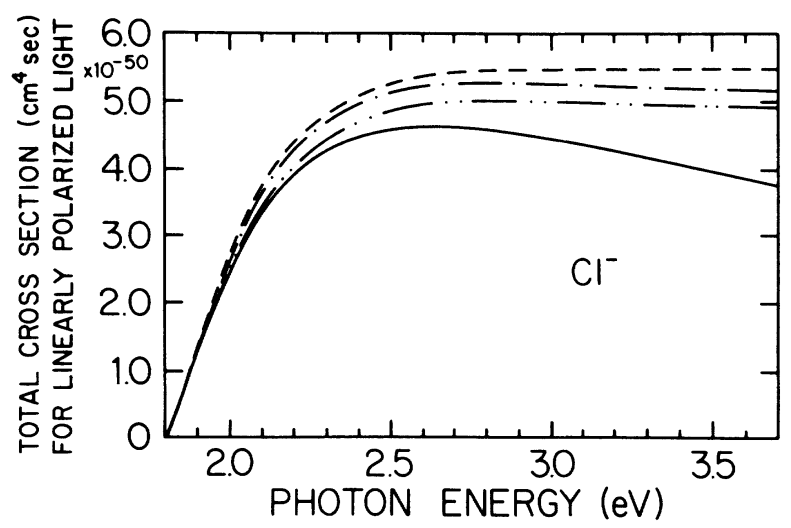

FIG. 5. Generalized two-photon total cross section (length form) for the transitions $\mathrm{Cl}^{-} 3 p^{6}\left({ }^{1} S\right)+2 \gamma \rightarrow \mathrm{Cl} 3 p^{5}\left({ }^{2} P\right)+e^{-}$for linearly polarized incident photons with energies below the single-ionization threshold. Notations are the same as for Fig. 2.

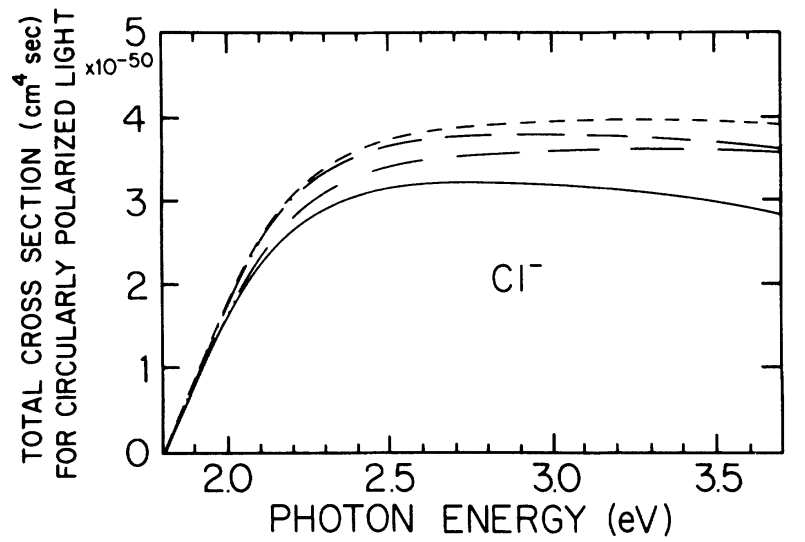

FIG. 6. Generalized two-photon total cross section (length form) for the transitions $\mathrm{Cl}^{-} 3 p^{6}\left({ }^{1} S\right)+2 \gamma \rightarrow \mathrm{Cl} 3 p^{5}\left({ }^{2} P\right)+e^{-}$for circularly polarized incident photons with energies below the single-ionization threshold. Notations are the same as for Fig. 2.

confirm these theoretically predicted electron correlation effects by measuring a modestly declining total cross section for either linearly or circularly polarized light and/or a (more modestly) declining ratio of linear to circular polarization cross sections in this energy region.

Finally, it is interesting to compare the present results with those for argon. ${ }^{2}$ The major differences, of course, are due to the different long-range fields experienced by the photoelectron. Thus the two-photon photodetachment cross sections are zero at threshold as contrasted with the finite value of the two-photon ionization cross sections in argon. Secondly, the absence of excited states in $\mathrm{Cl}^{-}$results in an absence of the resonance structures (and the consequent important role for intermediate-state interchannel interactions near these resonances) that are predicted for argon. ${ }^{2}$ Otherwise, however, the roles of each of the electron correlations we have studied, both in the percent magnitudes of their effects on the partial cross sections and in their relative importance with respect to one another, are very similar in both $\mathrm{Cl}^{-}$and in Ar.

\section{Comparisons with results of others}

The only experimental results on the two-photon detachment cross section of the negative chlorine ion of which we are aware are the relative measurements of Trainham et al. ${ }^{6}$ They measured the fraction of chlorine minus ions surviving irradiation by laser photons having energies from threshold to about $0.13 \mathrm{eV}$ above. Over this region their results confirm the Wigner threshold behavior $^{27,28}$ of the detachment cross section, as do our predictions. Hence these measurements are not high enough in energy above threshold to confirm the electron correlation effects we predict.

Crance $^{7}$ has recently calculated independent electron model two-photon detachment cross sections for chlorine minus. Her results for the total cross section for linearly polarized light, shown in Fig. 7 together with our results, 


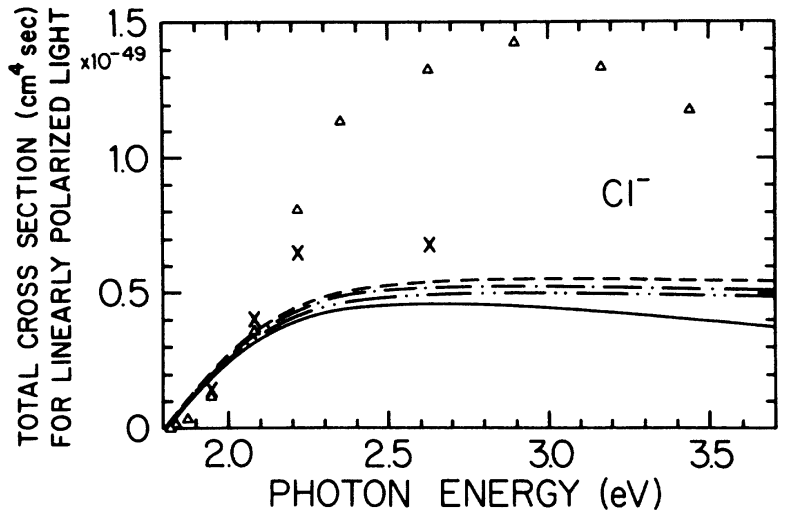

FIG. 7. Generalized two-photon total cross section (length form) for the transitions $\mathrm{Cl}^{-} 3 p^{6}\left({ }^{1} S\right)+2 \gamma \rightarrow \mathrm{Cl} 3 p^{5}\left({ }^{2} P\right)+e^{-}$for linearly polarized incident photons. The triangles are the frozen-core free-electron approximation results of Crance (Refs. 7 and 29), the crosses are the frozen-core Hartree-Fock results of Crance (Ref. 7). The curves are present results (see Fig. 2 for notation).

are carried out in two approximations. In the first, indicated by the triangles, the photoelectron wave function is that for a free electron. In the second, indicated by the crosses, the photoelectron wave function is a HartreeFock wave function. In both cases the $\mathrm{Cl}^{-}$ion initial state and the $\mathrm{Cl}$ atom final state employ the Hartree-Fock orbitals of the $\mathrm{Cl}^{-}$configuration, i.e., a frozen-core approximation is employed. Both of Crance's results lie noticeably below ours just above threshold, where the Wigner threshold law ${ }^{27,28}$ applies. Beyond $0.4 \mathrm{eV}$ above threshold, however, they lie substantially above all our results, with the free-electron cross sections ${ }^{29}$ lying a factor of 3 higher at $\hbar \omega \cong 2.9 \mathrm{eV}$. Crance's HF results, of course, are much closer to our own HF results, indicated by the dashed line. Our HF wave functions are also calculated in the frozen-core approximation and employ the $L S$-dependent $V^{N-1}$ potential ${ }^{21}$ for the photoelectron. However, the reasons for the significant differences in the two HF-level calculations, particularly for the results at higher energies, are unclear.

Prior to the very recent calculations of Crance, ${ }^{7}$ the only other results for the two-photon detachment cross section of $\mathrm{Cl}^{-}$of which we were aware was the central potential model calculation of Robinson and Geltman. ${ }^{9}$ These results, shown in Fig. 8, are more than a factor of 2 above our results at $\hbar \omega \cong 2.2 \mathrm{eV}$. Since the Robinson and Geltman results included a long-range polarization potential in their calculation, we decided to see what effect on our results such a potential would have. We thus performed additional calculations including in the equations for the photoelectron the following polarization potential:

$$
U(r)=\frac{-\alpha\left[1-\exp \left(-r / r_{p}\right)\right]}{\left(r^{2}+r_{p}^{2}\right)^{2}}
$$

For this potential we employed the same value of the cutoff parameter $r_{p}$ as in Ref. $9, r_{p}=2.5$ a.u. However,

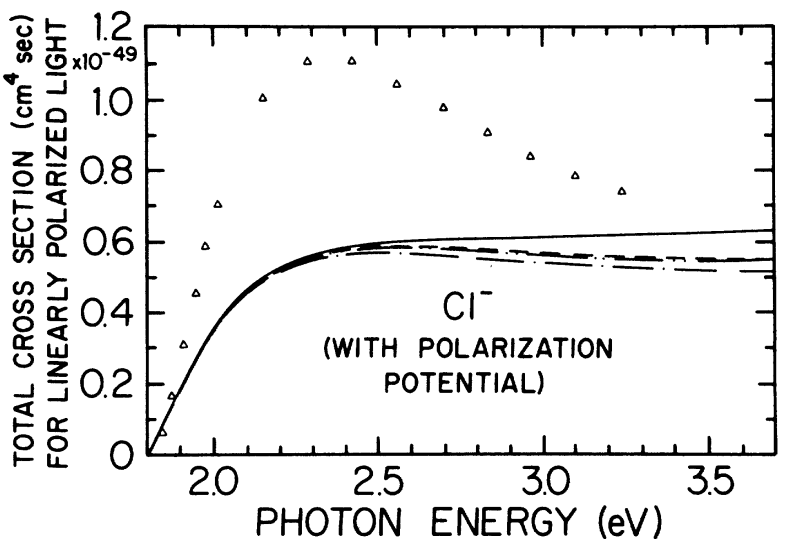

FIG. 8. Generalized two-photon total cross section (length form) for the transitions $\mathrm{Cl}^{-} 3 p^{6}\left({ }^{1} S\right)+2 \gamma \rightarrow \mathrm{Cl} 3 p^{5}\left({ }^{2} P\right)+e^{-}$for linearly polarized incident photons. The triangles are the central potential model calculations, including a long-range polarization potential, of Robinson and Geltman (from Table II of Ref. 9). The curves are the present results including the longrange polarization potential in Eq. (20) in the calculation of the wave functions for the photoelectron (see Fig. 2 for notation).

for the average static dipole polarizability $\alpha$ we employed the more accurate value of Reinsch and Meyer, ${ }^{30}$ $\alpha=14.71$ a.u., which is one-third smaller than the value employed in Ref. 9.

As shown in Fig. 8, even with our inclusion of a longrange polarization potential, the central potential model results of Robinson and Geltman ${ }^{9}$ still lie more than a factor of 2 above our results around $\hbar \omega \cong 2.2 \mathrm{eV}$. They are also significantly peaked, as compared to the plateau predicted by our calculations. Inclusion of a long-range polarization potential completely changes the effects of electron correlations in our calculations, resulting, for example, in the ESI results lying above our HF results. Also, the cross sections with the polarization potential are somewhat larger than those without it, as one might expect for an attractive potential.

We emphasize, however, that we do not regard our results which include polarization effects, shown in Fig. 8, as preferable to those in Figs. 5 and 7, which do not include polarization effects. This is because no accounting was made of the change in the core electron wave functions due to the polarization of the core by the photoelectron. This effect may be treated approximately by including a core-polarization correction to the dipole operator. ${ }^{31}$ We have not done this for three reasons. Firstly, we prefer to treat all electron correlation effects by $a b$ initio rather than semiempirical procedures. Secondly, our primary purpose in introducing the same kind of longrange polarization potential into our calculation as done by Robinson and Geltman was to see if that potential was the primary reason for the large discrepancy between our two results for the two-photon detachment cross sections. We have found it is not. Thirdly, recent calculations ${ }^{32}$ for the single-photon detachment cross sections of the negative halogen ions have achieved good agreement with 
experiment without any treatment of polarization effects. This seems to indicate the insignificance of polarization effects for chlorine minus, at least for the present level of accuracy of experimental measurements.

\section{CONCLUSIONS}

We have presented theoretical calculations of the twophoton detachment cross sections for the chlorine negative ion using a transition matrix method. We have also analyzed in detail the influence of a number of electron correlation effects on these cross sections. Our results indicate that electron correlations produce a $25-30 \%$ decrease in the total two-photon detachment cross sections just below the single-photon ionization threshold as compared to predictions obtained using the Hartree-Fock (HF) approximation. Furthermore, the theoretical predictions for the total cross sections which include electron correlation effects tend to decrease modestly with increasing photon energy above about $\hbar \omega=2.5 \mathrm{eV}$, whereas the HF level predictions are rather flat. These differences in the shape of the cross sections makes it easier for experiment to confirm the presence of these electron correlation effects.

A number of comparisons have been made of our results with results of other groups. In agreement with the experimental results of Trainham et al. ${ }^{6}$ we find that the
Wigner threshold law $\mathrm{w}^{27,28}$ is obeyed just near threshold. The experimental results do not extend to high enough energy above threshold, however, to test our predictions for the cross-section shape beyond the region of applicability of the Wigner threshold law. In comparison with the central potential model calculations of Robinson and Geltman $^{9}$ and the free-electron calculation of Crance ${ }^{7}$ our results give a cross section maximum which is a factor of 2 to 3 lower and a shape which is considerably flatter. In particular, our HF results are much closer to our fully correlated predictions than to either of these other calculations, ${ }^{7,9}$ thereby indicating the importance of starting with an accurate representation for the electron wave functions. Lastly, we do not expect long-range polarization effects to play an important role in the twophoton detachment process for the negative chlorine ion.

\section{ACKNOWLEDGMENTS}

We wish to thank Michèle Crance for providing us with the numerical values for her two-photon calculations for the negative chlorine ion. We thank also David Norcross and Cheng Pan for helpful discussions concerning polarization effects on photoionization and photodetachment cross sections. This work was supported in part by National Science Foundation Grant No. PHY8601429.
"Present address: Department of Chemistry, University of Kansas, Lawrence, KS 66045.

${ }^{1}$ A. F. Starace, Phys. Scr. T17, 221 (1987).

${ }^{2}$ A. F. Starace and T. F. Jiang, Phys. Rev. A 36, 1705 (1987).

${ }^{3}$ A. L'Huillier and G. Wendin, J. Phys. B 20, L37 (1987).

${ }^{4}$ A. L'Huillier and G. Wendin, Phys. Rev. A 36, 4747 (1987).

${ }^{5}$ M. S. Pindzola and H. P. Kelly, Phys. Rev. A 11, 1543 (1975).

${ }^{6}$ R. Trainham, G. D. Fletcher, and D. J. Larson, J. Phys. B 20, L777 (1987).

${ }^{7}$ M. Crance, J. Phys. B 20, 6553 (1987).

${ }^{8}$ A. L'Huillier and G. Wendin, J. Phys. B 21, L247 (1988).

${ }^{9}$ E. J. Robinson and S. Geltman, Phys. Rev. 153, 4 (1967).

${ }^{10}$ A. F. Starace and T. F. Jiang, Bull. Am. Phys. Soc. 32, 1278 (1987).

${ }^{11}$ T. N. Chang and U. Fano, Phys. Rev. A 13, 263 (1976); 13, 282 (1976).

12 A. F. Starace and S. Shahabi, Phys. Rev. A 25, 2135 (1982).

${ }^{13}$ A. Dalgarno and J. T. Lewis, Proc. R. Soc. London, Sect. A 233, 70 (1955).

${ }^{14}$ C. Froese-Fischer, Comput. Phys. Commun. 4, 107 (1972); The Hartree-Fock Method for Atoms (Wiley, New York, 1977).

15J. R. Swanson and L. Armstrong, Jr., Phys. Rev. A 15, 661 (1977); 16, 1117 (1977).

${ }^{16}$ A. L'Huillier, L. Jönsson, and G. Wendin, Phys. Rev. A 33, 3938 (1986).

${ }^{17}$ A. F. Starace, in Handbuch der Physik, edited by W. Mehlhorn (Springer, Berlin, 1982), Vol. 31, pp. 1-121.

${ }^{18}$ A. F. Starace, in Fundamental Processes in Energetic Atomic
Collisions, edited by H. O. Lutz, J. S. Briggs, and H. Kleinpoppin (Plenum, New York, 1983), pp. 69-110.

${ }^{19}$ B. W. Shore and D. H. Menzel, Principles of Atomic Spectra (Wiley, New York, 1968), p. 340. Note that in Eq. (19) of Ref. 2 the second and third arguments of the Slater integrals should be switched in order to be consistent with the definition given by Shore and Menzel.

${ }^{20}$ C. Froese-Fischer, Comput. Phys. Commun. 1, 151 (1969); 4, 107 (1972).

${ }^{21}$ See Ref. 17, p. 73, and references therein.

${ }^{22}$ R. T. Pu and E. S. Chang, Phys. Rev. 151, 31 (1966).

${ }^{23}$ H. J. Silverstone and M. L. Yin, J. Chem. Phys. 49, 2026 (1968).

${ }^{24}$ S. Huzinaga and C. Arnau, Phys. Rev. A 1, 1285 (1970).

${ }^{25}$ A. Burgess, Proc. Phys. Soc. London 81, 442 (1963).

${ }^{26}$ P. Lambropoulos, Adv. At. Mol. Phys. 12, 87 (1976).

${ }^{27}$ E. P. Wigner, Phys. Rev. 73, 1002 (1948).

${ }^{28}$ A.R. P. Rau, Comments At. Mol. Phys. 14, 285 (1984).

${ }^{29}$ Note that the free-electron results shown in our Fig. 7 were obtained by Crance using the Dalgarno-Lewis method (Ref. 13) to sum over intermediate states. According to Crance (private communication) these results differ by about $5 \%$ from the more approximate results published by her in Ref. 7.

${ }^{30}$ E. A. Reinsch and W. Meyer, Phys. Rev. A 14, 915 (1976).

${ }^{31}$ D. W. Norcross, Phys. Rev. A 7, 606 (1973), and references therein.

${ }^{32}$ V. Radojević, H. P. Kelly, and W. R. Johnson, Phys. Rev. A 35, 2117 (1987). 